\title{
Mathematical Objects arising from Equivalence Relations, and their Implementation in Quine's $\mathrm{NF}$
}

\author{
Thomas Forster \\ Department of Pure Mathematics and Mathematical Statistics, \\ University of Cambridge
}

January 4, 2014

\begin{abstract}
Many mathematical objects arise from equivalence classes, and invite implementation as those classes. Set existence principles that would enable this are incompatible with ZFC's unrestricted aussonderung but there are set theories (e.g. NF and Church's CUS) which admit more instances than does ZF. NF provides equivalence classes for stratified relations only. Church's construction provides equivalence classes for "low" sets, and thus - e.g. - a set of all (low) ordinals. However that set has an ordinal in turn, which is not a member of the set constructed, so no set of all ordinals is obtained thereby. This "recurrence problem" is discussed.

This essay can be seen as a non-technical companion to [6] and is, like it, a precursor to - and will be subsumed into - the longer book-length treatment of these matters that I have promised myself I will write. ${ }^{1}$
\end{abstract}

\section{Introduction}

I would like to start off by insisting on some terminology: what we try to do to cardinals, ordinals (and other mathematical entities) when we come to set theory is not to define them but to implement them. We don't need to define cardinals: we know perfectly well what a cardinal is: a cardinal is that thing that two sets have in common (i.e., to which they are related in the same way) precisely when they are equinumerous. If we wish to tell a story in which everything is a set then we have to have ways of implementing these mathematical objects from outside set theory as sets. Inattention to the distinction between definition and implementation can result in absurdities. For example, with the

\footnotetext{
${ }^{1}$ I am very grateful to Roy Cook and Erich Reck for the invitation to give a talk under this title at their thoroughly enjoyable and instructive workshop, and for the opportunity it provided me to get my thoughts in order.
} 
von Neumann implementation of cardinals and ordinals into set theory it happens that the three distinct mathematical objects (i) the ordinal $\omega$, (ii) the set $\mathbb{N}$ of natural numbers, and (iii) the cardinal number $\aleph_{0}$ are all implemented as the same set. (The set itself has a purely set-theoretical characterisation as the set of wellfounded hereditarily transitive finite sets). These three mathematical objects are all distinct, and-however convenient it may be to implement them in set theory by the same sets - it would make no sense to attempt to define them to be the same. It's the same mistake as trying to define $\pi$ to be $22 / 7 .^{2}$

This importance of this distinction is surely one of the morals one can draw from [1]. Benacerraf's point about the Zermelo naturals and the von Neumann naturals is that they can't both be a correct account of what natural numbers are; what he doesn't say - but what thoughtful readers can work out for themselves - is that they can nevertheless both be acceptable implementations of natural numbers. There is a story one could tell about the emergence of the concept of implementation (nowadays folklore among computer scientists), about its roots in mathematics, and about how it can feed back into Philosophy of Mathematics and into mathematical praxis; it should be told.

Confusing definition with implementation does more than merely annoy pedants. It can - and all too often does - lead people into the error of thinking that they have proved something about a suite of entities when the only thing they have actually proved is the corresponding fact about the sets that implement those entities. For example, I have seen it claimed that, given that the order relation on (von Neumann) ordinals is $\in$, then the wellfoundedness of the order relation on ordinals follows easily from the axiom of foundation (the axiom that says that $\in$ is wellfounded). By resolving to use the word 'implement' instead of 'define' we arm and protect ourself against such craziness and we set off on the right foot.

To implement a suite of mathematical objects into set theory is to provide an interpretation $\mathcal{I}$ from a theory $T$ of those objects into a theory $T^{\prime}$ of set theory. In recent years logicians have become increasingly interested in studying interpretations between theories as mathematical objects in their own right ${ }^{3}$, not least for what they can tell us about relations between theories.

One notion that has emerged is that of synonymous theories. Synonymy

\footnotetext{
${ }^{2}$ While I am on this subject I'd like to draw my readers' attention to the strange circumstance that, if we amplify the von Neumann intepretation by the device (which Erich Reck tells me goes back at least to Dedekind) of thinking of integers as equivalence classes of ordered pairs of naturals in the familiar way, then the integer 0 is the same set as the identity relation on $\mathbb{N}$; the integer -1 is the successor relation on $\mathbb{N}$; every integer is a binary relation, and-in a curious echo of Church numerals in $\lambda$-calculus - addition of integers is relational composition! This is tolerable if we are thinking of these sets as implementations of the mathematical entities under consideration, but if integers really are sets, then the integer 0 really would be the same thing as the identity relation on $\mathbb{N}$, and this is surely a reductio ad absurdum. If the integer 0 is the same thing as the identity relation on $\mathbb{N}$ then we can surely all stop worrying about whether or not the number 1 is Julius Cæsar (It is —of course! - the predecessor relation on $\mathbb{N}$.)

${ }^{3}$ My Doktorvater Adrian Mathias used to say that a logician is that kind of mathematician that thinks that a formula is a mathematical object.
} 
of two theories is a very strong kind of mutual interpretability. Roughly, two theories are synonymous iff they have the same models. A standard example is Boolean rings and Boolean algebras. A good place to start is with [10]. Synonymy will crop up here, later.

\section{NF}

The brief the organisers gave me specifically mentioned NF and natural numbers, and the two are joined in this context because of the possibility held out by NF (being a set theory with a universal set) of implementing natural numbers in a - natural! - way as equipollence classes. It has been felt since the days of Russell and Frege that this is a natural way to implement entities arising from equivalence relations.

This is a fruitful restriction, but a restriction nonetheless, and in several ways: NF is not the only set theory that allows a universal set, since there are also CUS and Positive Set Theory. CUS (see [3]) and its variants resemble $\mathrm{NF}$ in allowing large sets and in allowing the implementation of cardinals and suchlike as equivalence classes. At present it is still unclear whether or not NF is consistent $^{4}$, but we do at least know that NFU is consistent, and it does all the good things NF does about large sets, thereby facilitating the implementation of mathematical objects as equivalence classes.

The second restriction is to entities arising from equivalence relations. It is worth noting en passant that not all entities in need of implementation arise from equivalence relations. Typically, a formal theory - of widgets, as it might be - will need to be able to quantify over ordered pairs of widgets. And there is no obvious way of thinking of ordered pairs as equivalence classes

The third restriction is that even those mathematical entities that do arise from equivalence relations do not have to be implemented as equivalence classes, or even as restrictions of them: the obvious example is the von Neumann implementation of ordinals in $\mathrm{ZF}(\mathrm{C})$ as hereditarily transitive sets.

Nevertheless we are going to restrict ourselves to entities arising from equivalence relations... and not only because such entities are the easiest to discuss, but also because they seem to be the chief preoccupation of the neologicists.

Many mathematical entities arise from equivalence relations, and it is natural to feel that such entities should in principle be implementable in set theory as equivalence classes. Obvious examples are cardinals (equivalence classes under equipollence), ordinals (isomorphism classes of wellorderings); an (abstract) group arises from an isomorphism class of concrete groups; points-at-infinity arise from the equivalence relation "parallel-to" on lines. One could go on. As mentioned above, NF set theory has the pleasing feature that most mathematical entities arising from equivalence classes can in fact be implemented in NF straightforwardly as those equivalence classes. In theories with unrestricted separation (such as Zermelo and Zermelo-Fraenkel) the equivalence classes cannot

\footnotetext{
${ }^{4}$ though the situation may well have changed by the time this document goes to the printer.
} 
be sets because they would lead to a universal set and thence-by unrestricted separation $^{5}$ - to the Russell Class. Things work in NF because NF does not have unrestricted separation (it has separation only for stratified formulæ) but it does have comprehension for stratified formulæ- which Zermelo and ZF do not. NF thus lacks unstratified separation, but does have stratified comprehension. Swings and roundabouts.

"Stratified"? A formula of set theory is stratified iff by assigning numerical type subscripts to its variables we can turn it into a wff of simple type theory. That is to say, a wff $\phi$ is stratified iff we can find a stratification assignment ('stratification' for short) for it, namely a map $f$ from its variables (after relettering where appropriate) to $\mathbb{N}$ such that if the atomic wff ' $x=y$ ' occurs in $\phi$ then $f\left({ }^{\prime} x '\right)=f\left({ }^{\prime} y\right.$ '), and if ' $x \in y^{\prime}$ occurs in $\phi$ then $f\left({ }^{\prime} y\right.$ ' $)=f\left({ }^{\prime} x\right.$ ' $)+1$. (NB: I really do mean 'variable' not 'variable-occurrence': all occurrences of any one variable must receive the same natural number under the stratification assignment; without this additional restriction every formula in the language of set theory would be stratified!). Variables receiving the same integer in a stratification are said to be of the same type. Another way of thinking about stratification is to say that a formula of the language of set theory is stratified iff it could become a formula of the language of type theory by decorating its variables with integer subscripts.

We also need the notion of a homogeneous formula. A formula is homogeneous iff it is stratified and additionally all its free variables are of the same type. Thus ' $x=y$ ' is homogeneous but ' $x \in y$ ' is not, altho' it is stratified. We can define the notions of stratified relation and stratified function and of homogeneous relation and homogeneous function in the obvious way. The significance of this notion of homogeneity is that if $F$ is a homogeneous relation then NF proves that the graph of $F$ is a set. Further the closure of a set under a homogeneous function is also a set. Mere stratification is not sufficient. Thus the graph of $\in$ (which is stratified but inhomogeneous) is not a set, and the transitive closure of a set (its closure under $\epsilon$ ) is not always a set. Nevertheless the "skewed-membership" relation $\{\langle\{x\}, y\rangle: x \in y\}$ is a set, and it contains in some sense the "same" information. This phenomenon of sethood of "skewed" versions of things that cannot themselves be sets is frequently encountered, and is very important.

NF is now axiomatised by Extensionality plus a comprehension scheme for stratified expressions: $\{x: \phi(x, \vec{t})\}$ (parameters are allowed) is a set. It cannot be emphasised too loud or too often that NF is a one-sorted set theory: the variables in the formulæ do not have types; they are given types only while one is checking their host formula to see whether or not it is stratified (tho' one can-and does - say that, in ' $x \in y$ ', ' $x$ ' has a type one lower than the type of ' $y$ '). At no stage do the sets of which NF talks to us ever have types.

As the reader can see, it is easy enough to explain what the stratification condition on formulæ is, but it's rather more difficult to explain what it means

\footnotetext{
${ }^{5}$ Separation is the principle that every subcollection of a set is a set.
} 
deep down. It certainly looks like a mere syntactic trick, but there is a completeness theorem that provides a semantic motivation: it turns out that stratified expressions are precisely those preserved by all applications of a construction known as Rieger-Bernays permutation models ${ }^{6}$. That much is fairly straightforward. It also turns out - more significantly but also more obscurely - that all (or nearly all) mathematical notions can be implemented into set theory in such a way that the relations-between-sets that implement the relations-between-theoriginal-mathematical entities get captured by stratified expressions - and those that can't are in some interesting but obscure sense special. It's not entirely clear how to express this fact, it's less clear what its significance is, and even less clear still whether these two facts (the completeness theorem and the implementability) make stratification an appropriate criterion for set existence. After all, the fact that stratification is important and natural doesn't obviously make it the correct criterion for a formula to have an extension, for its real significance may lie elsewhere.

Stratification arises fairly straightforwardly from a syntactic device of Russelland-Whitehead that is designed to forestall Russell's paradox. However there is also an endogenous typing in Mathematics which on the face of it has nothing to do with set theory. It found its way from mathematics into the theory of programming languages, where it is commonplace to have a typing discipline that distinguishes naturals from floating-point reals, and both of them from booleans and arrays. The striking fact (and I am trying to write a book about this) is how often these two notions of typing coincide in practice and how well they fit.

Until that book is written I shall restrict myself to the observation that what this neat fit means for NF studies is that, for any naturally occurring kind of mathematical entity (at least those which arise from equivalence classes) there will be an NF-implementation such that

(i) the entities themselves (the tokens) are implemented as sets;

(ii) the collection of all of the tokens is implemented as a set, and

(iii) (at least some of) the natural operations on them are captured by homogeneous formulæ and their graphs implemented as sets.

(The weasel words in brackets in (iii) are needed because of complications with quotients; for example: " $G_{3}$ is the quotient of $G_{1}$ over its normal subgroup $G_{2}$ " is stratified but not homogeneous.)

In particular the three expressions in the language of set theory that say

(a) " $x$ and $y$ are equinumerous";

(b) " $x$ is the set of all things equinumerous with $y$ "; and

(c) " $x$ is an equinumerosity class"

\footnotetext{
${ }^{6}$ This result (see [5]) is very much in the spirit of the results in classical model theory that say things like: universal sentences are precisely those preserved under substructure; universal existential sentences are precisely those preserved under colimits.
} 
are all stratified - and (a) is homogeneous. The fact that (a) is homogeneous implies that - in NF - the graph of the equinumerosity relation is a set; the fact that (b) is stratified implies that every (Frege) cardinal is a set (i.e., every set has a Frege cardinal and that cardinal is a set) and the fact that (c) is stratified implies that the collection of all (Frege) cardinals is a set. Observe that ' $y=|x|$ ' (" $y$ is the cardinal of $x$ ") is stratified. However the type of ' $x$ ' is one less than the type of ' $y$ ', so ' $y=|x|$ ' is not homogeneous. This echoes the fact that if $y$ is the cardinal of $x$ then $x$ and $y$ are objects (prima facie) of different abstract data types. In contrast " $x$ and $y$ are cardinals and $x<y$ " is homogeneous. It turns out (I shall spare the reader the technical details) that all the usual operations on natural numbers turn out to be homogeneous.

\section{The Recurrence Problem}

Despite what was said above about how Mathematics can be implemented into set theory in a stratified way, complications can arise if it happens that the flavour of mathematical entity whose implementation we are discussing (as it might be widgets) has the feature that the family of isomorphism classes of widgets supports a widget structure. This widget structure of the family of widgets can result in stratification conflicts. There are many examples of this kind of thing, and Feferman writes about them in [4] (tho' not in terms of stratification conflicts). The collection of all categories supports a category structure; the family of all [isomorphism classes of] semigroups supports a semigroup structure, namely direct product; the family of wellfounded binary structures supports a wellfounded relation, namely end-extension; and so on. In [4] this phenomenon is mentioned as a motivation for developing a foundation in which one can talk about universal objects: the set of all groups, of all cardinals, and so on. The best and simplest illustration of what one might call the recurrence problem ${ }^{7}$ (as well as being the one most pertinant to us at this Frege workshop) comes from cardinal arithmetic - specifically the arithmetic of $\mathbb{N}$, the natural numbers.

Natural numbers count [multiplicities/collections of] concrete objects - at any rate in the first instance it's concrete objects that they count. But once natural numbers have appeared, we have the possibility of counting [multiplicities/collections of] natural numbers. Can we count these new collections with the same natural numbers used to count concrete objects? Or does that counting require a novel abstract data type of a new kind of number? (Sheep farmers in the Pennines use distinctive numerals - which are in fact $p$-celtic words - for counting sheep, rather than the ordinary english words they use for counting everything else. I have not been able to find a linguistics literature on the possibility of using different suites of counting-words for objects of different flavours.) The answer will depend on how squeamish you are. Computer scientists are used to a situation where two distinct types of widget and wombat will give rise to the two different types of widget-list and wombat-list; we say of the constructor

\footnotetext{
${ }^{7}$ I've just invented this expression, in case you were wondering why you'd never seen it before.
} 
list that it is polymorphic. However the output of the length function applied to lists is always taken to be monomorphic: the same kind of number is used to measure both the length of lists-of-widgets and the length of lists-of-wombats. The polymorphism of lists is lost in the progression to natural numbers. Most of us are happy with monomorphic natural numbers. It seems natural enough: two multiplicities have the same cardinal iff there is a bijection between them, and there seems to be no reason why two multiplicities of different types should not have a bijection between them. And, indeed, the natural numbers of NF are monomorphic. However there is a ghost of the primordial polymorphism still tangible in NF. The cardinal number of $\{m: m<n\}$ (where $n$ is a given natural number) is a perfectly ordinary natural. So there is a function sending the natural number $n$ to the natural number $|\{m \in \mathbb{N}: m<n\}|$. Specker called this function $T: T n=|\{m: m<n\}|$ for $n$ a natural number. ' $m=T n$ ' is stratified but inhomogeneous, and the graph of $T$ is not provably a set. Rosser's axiom of counting from [8] says - in NF - that this function is the identity, and we now know that Rosser's counting principle is not a theorem of NF. Outside $\mathrm{NF}$ it says that among the sets belonging to a natural number $n$ is the set $\{m: m<n\}$ of natural numbers smaller than $n$.

The idea that - more generally - each ordinal counts the set of its predecessors [in their natural order] seems to be in Cantor [2], indeed to be coeval with the idea of ordinal itself.

The recurrence problem turns up also in CUS. The Grand Scheme with CUS is to construct from a given model of ZF a new model with big sets while maintaining the wellfounded sets of the new model as a model of ZF-in fact an isomorphic copy of the original model. CUS can be axiomatised by (i) complementation: every set has a complement; (ii) The wellfounded sets are a model for ZF; (iii) replacement holds for wellfounded sets (the image of a wellfounded set in a function is a set) and finally a scheme of existence for $\{x: x \sim y\}$ whenever $y$ is a wellfounded set and $\sim$ is a kind of generalised equipollence relation to be specified. See [3] for details. Sets (in the new model) that are the same size as wellfounded sets (in the new model) are said to be low. In Church's original construction he provides a universal set. Sheridan [9] shows how to add the singleton function as a set. Church shows how, for more-or-less any equivalence relation $\sim$, to add $\sim$-equivalence classes for low sets. (Equivalence classes for non-low sets are not provided.) He explicitly does this for equipollence (obtaining cardinals - and also generalisations that he calls $j$-cardinals) and, altho' he doesn't do it for arbitrary equivalence relations, it is clear that he knows how to. There is some suggestion (for example at the end of [7]) that the theories satisfied by the models furnished by these constructions are synonymous with the theory of the model that is operated on. If correct, this would account for the comparative straightforwardness of the constructions that give us the universal set, the singleton function, the $j$-cardinals of low sets and so on, for that straightforwardness would be a reflection of the fact that these new entities are merely pointless epiphenomena with no mathematical content. But that is yet to be established. 
However it is significant that the construction generally does not provide global equivalence classes, but merely equivalence classes of low sets. And the difficulty we experience in providing global equivalence classes seem to arise only where there is a recurrence problem. Why does recurrence cause a problem? Suppose the family of all equivalence classes of widgets supports a widget structure. One adds - by a Church-style coding trick - equivalence classes of the widgets in one's field of view (i.e., the low widgets). But then (in consequence of the Church construction) there are now new widgets, and they might not all be low and so might not fit into the equivalence classes so far provided. So one repeats the construction... with potentially the same unsatisfactory result. There doesn't seem to be any global argument that would tell us that this process should reach a fixed point. Thus it comes about that no known enhancement of Church's construction gives us a model containing a set of all ordinals. NF alleges that there is such a set, and thus in effect assumes that the recurrence problem has been solved. No wonder the consistency problem for NF is so hard.

\section{Burali-Forti}

The most notorious example of the recurrence problem is undoubtedly the Burali-Forti paradox. Recall that an ordinal is a mathematical object associated with a wellordering: every wellordering has an ordinal, and two wellorderings have the same ordinal iff they are isomorphic. One can think of ordinals as equivalence classes of wellorderings (which is how they fall under the rubric of this essay) and that indeed is precisely how they are implemented in NF.

The Burali-Forti paradox arises because every set of ordinals is naturally wellordered by $<_{O n}$, the order relation on ordinals, and so must have an ordinal. Notorious it may be, but its notoriety has not resulted in it receiving close attention - rather in it being avoided and - in consequence - remaining poorly understood .... and certainly never mentioned in front of the children. The elephant in the drawing room? Wrong elephant.... It's more like the elephant in the old Indian trope about the five blind men. You have to approach it from more than one point of view if you are to have any hope of understanding what is going on.

In the hope of obtaining some enlightenment, and on the assumption that the reader is familiar with the ZF analysis of Burali-Forti, let me concentrate on an analysis from an NF perspective.

Let us take as our point of departure the idea that natural numbers are prima facie polymorphic - and indeed that ordinals, too, are prima facie polymorphic. That is to say, when widget and gadget are different types we not only distinguish (as we must) between the two types widget-list and gadget-list, we even distinguish between length-of--widget-list and length-of--gadget-list, even though both these types are flavours of natural number! Our point of departure is to behave like Pennine sheep-farmers. Our first move is to identify, to coalesce into one type all these types that look like natural numbers. In particular we are going to regard the numbers-that-count-sets of [whatever] as 
the same types as the numbers that-count-sets-of-numbers-that-count-sets-of[whatever]. Our second move is to say further that, not only is $|\{n: n<x\}|$ a number of the same type as $x$, but that it is actually identical to $x$. This is Rosser's Counting Principle. Initially at least we perform this coalescence only on types of natural numbers (= finite ordinals) - it does seem to be safe there. However we can contemplate (a third move of) extending it to the ordinals (I suppose one should then call it Rosser's extended Counting Principle) and this is when the trouble starts.

I emphasised earlier that NF is a one-sorted theory: in particular it has only one type of ordinal. We can in any case prove from first principles that every set of ordinals (and there is now only one kind of ordinal) is wellordered by the comparative-magnitude relation for ordinals (which we can write ' $<_{O n}$ '), so in particular every initial segment $S$ of the ordinals is wellordered by $<_{O n}$, and now Rosser's Extended Counting Principle will tell us - if we let it - that the order type of $S$ is the least ordinal not in it.

NF tells us that the collection $N O$ of all ordinals is a set, and is wellordered by $<_{O n}$. And its length? If Rosser's Extended Counting Principle were to be believed then the length would have to be the first ordinal not in $N O$, and there is of course no such thing. Clearly Rosser's Extended Counting Principle fails for at least some big ordinals.

What does this failure mean for NF? We must not forget that NF is a onesorted theory, so we cannot go back to our state-of-nature in which ordinals were polymorphic; the effect is rather that-sometimes - where we would expect to find a single ordinal we instead find a whole hall of mirrors of them. For example: $N O$-ordered-by- $<_{O n}$ has an ordinal, which we shall call ' $\Omega_{0}$ '. What about the ordering of the ordinals below $\Omega_{0}$ ? Rosser's Extended Counting Principle would lead us to expect that its order type should be $\Omega_{0}$, but it evidently cannot be. It's a distinct ordinal, which I suppose we will have to call ' $\Omega_{1}$ ' ... and the ordering of the ordinals below $\Omega_{1}$ is of order type $\Omega_{2}$, and so on. Morally of course all these $\Omega_{n}$ belong to distinct abstract data types, but since NF is onesorted they are one and all compelled to belong to the one same type, so NF can express this insight only in a veiled or muted way. Dana Scott wrote to me, when I was a Ph.D. student, that NF is really a type theory, and it must have been this that he had in mind.

There are echoes here of the axiom of reducibility, but that is too difficult a topic for a brief note such as this.

\section{References}

[1] Paul Benacerraf "What Numbers Could not Be" The Philosophical Review 74 No. 1 (Jan., 1965), pp. 47-73.

[2] Georg Cantor, "Foundations of a General Theory of Manifolds" (1883)

[3] Church, A. "Set Theory with a Universal set". Proceedings of the Tarski Symposium. Proceedings of Symposia in Pure Mathematics XXV, ed. L. 
Henkin, Providence RI pp. 297-308. Also in International Logic Review 15 pp. 11-23.

[4] Solomon Feferman "Some formal systems for the unlimited theory of structures and categories" unpublished but available online: http://math.stanford.edu/ feferman/papers/Unlimited.pdf

[5] Forster, T.E. "Permutation Models and Stratified Formulæ, a Preservation Theorem". Zeitschrift für Mathematische Logic und Grundlagen der Mathematik 36 (1990) pp 385-388.

[6] Forster, T.E., "Implementing Mathematical Objects in Set theory". Logique et Analyse 50 No.197 (2007)

[7] Forster, T. E., "The Iterative Conception of Set". Review of Symbolic Logic. 1 (2008) pp 97-110.

[8] Rosser J.B. "Logic for Mathematicians" McGraw-Hill $195314+540$ pp.

[9] K.J. Sheridan "A Variant of Church's Set Theory with a Universal Set in which the Singleton Function is a Set" Logique et Analyse 572014.

[10] Albert Visser, "Categories of Theories and Interpretations", Logic in Tehran, Lect. Notes Log., vol. 26, Assoc. Symbol. Logic, La Jolla, CA, 2006, pp. 284341. also available at available at http://www.phil.uu.nl/preprints/preprints/PREPRINTS/preprint228.pdf

Department of Pure Mathematics and Mathematical Statistics

Centre For Mathematical Sciences

Wilberforce Road

Cambridge CB3 0WB

United Kingdom

tf@dpmms.cam.ac.uk 